\title{
IASI-NG: when improving our understanding of the Earth atmosphere requires to multiply innovations in an operational meteorological programme
}

\section{Adrien Deschamps}

Adrien Deschamps, "IASI-NG: when improving our understanding of the Earth atmosphere requires to multiply innovations in an operational meteorological programme," Proc. SPIE 11852, International Conference on Space Optics ICSO 2020, 1185204 (11 June 2021); doi: 10.1117/12.2599144

SPIE Event: International Conference on Space Optics - ICSO 2021, 2021, Online Only 


\section{International Conference on Space Optics-ICSO 2020}

Virtual Conference

30 March-2 April 2021

Edited by Bruno Cugny, Zoran Sodnik, and Nikos Karafolas
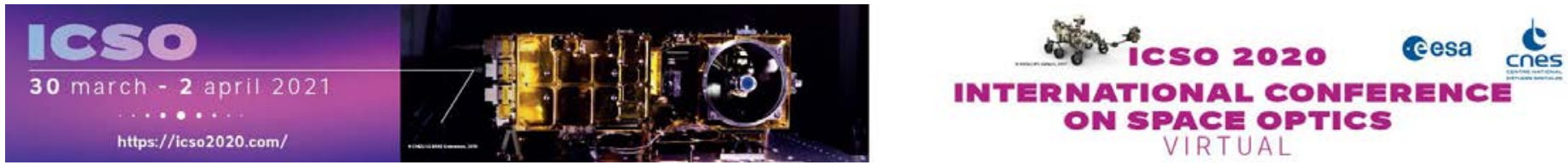

\section{IASI-NG: when improving our understanding of the Earth atmosphere requires to multiply innovations in an operational meteorological programme}

\section{Cesa




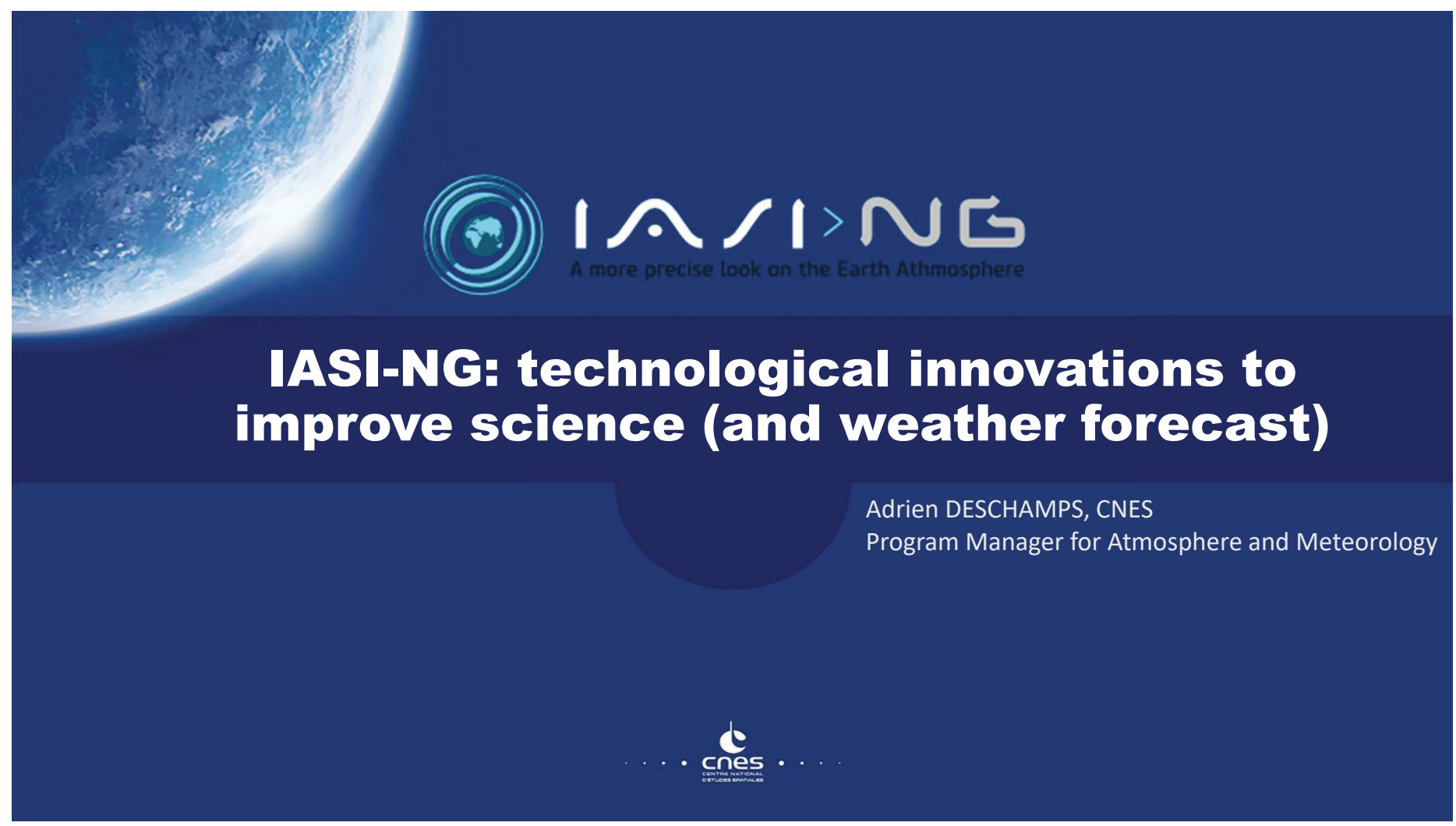

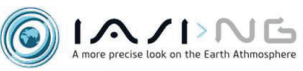

Innovation and space sciences in 2021

Do the space agencies - and industries - still accept to take risk for the development of space systems?

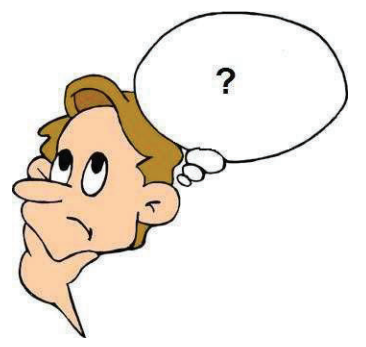

Do we accept risks only for nano-satellites or demonstrators ?

... the IASI-NG example partially answers the question... 


\section{The Earth Observation Program at CNES}

\section{CNES missions under development or in orbit}

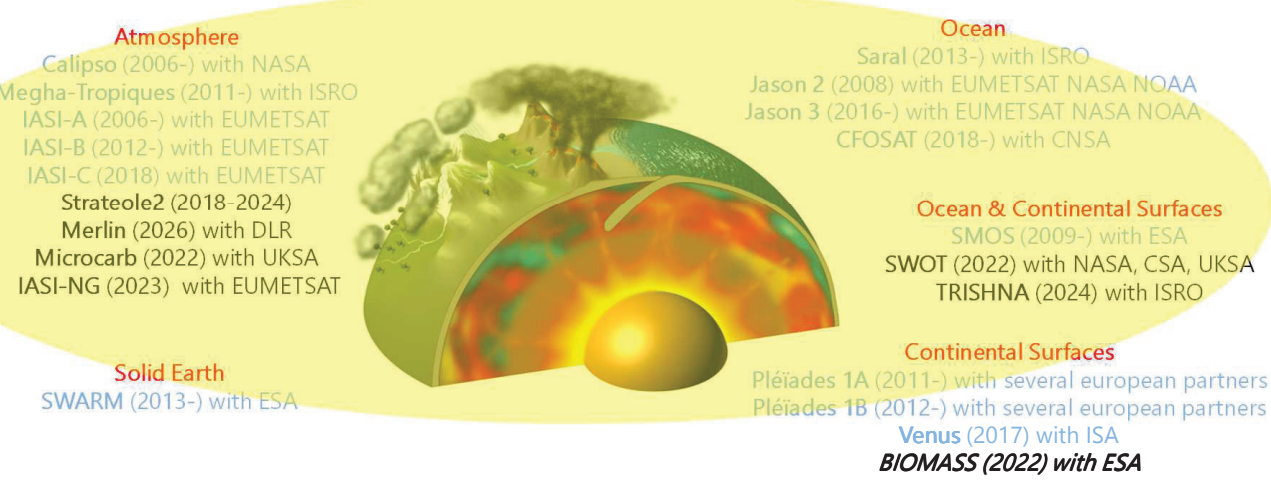

+ Contribution to Copernicus Sentinels : Sentinel 2, 3 and 6

$+\sim 10$ pre-phase A studies conducted every year $\rightarrow$ Preparation of the future

$\rightarrow$ A diversity of partners : NASA, EUMETSAT, DLR, ESA, CNSA, ISRO, ISA, ...

$\rightarrow$ All components of the Earth System are adressed, and their interactions

$\rightarrow$ Several multi-thematic missions : SMOS, SWOT, ...

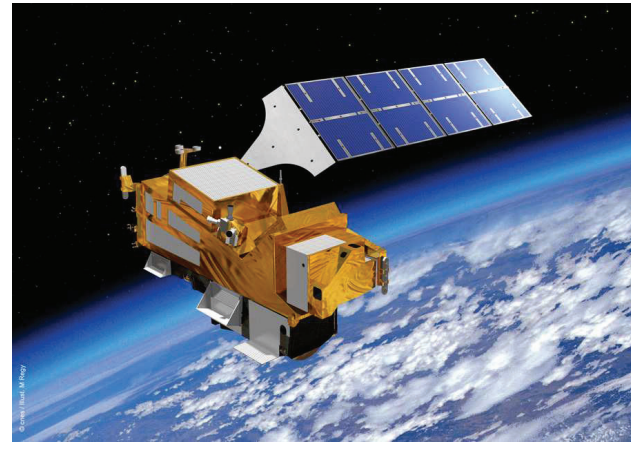

From IASI to IASI-NG: new challenges for ambitious scientific goals

Innovations from the instrumental concept to data processing

Some perspectives about future atmospheric sounding missions 


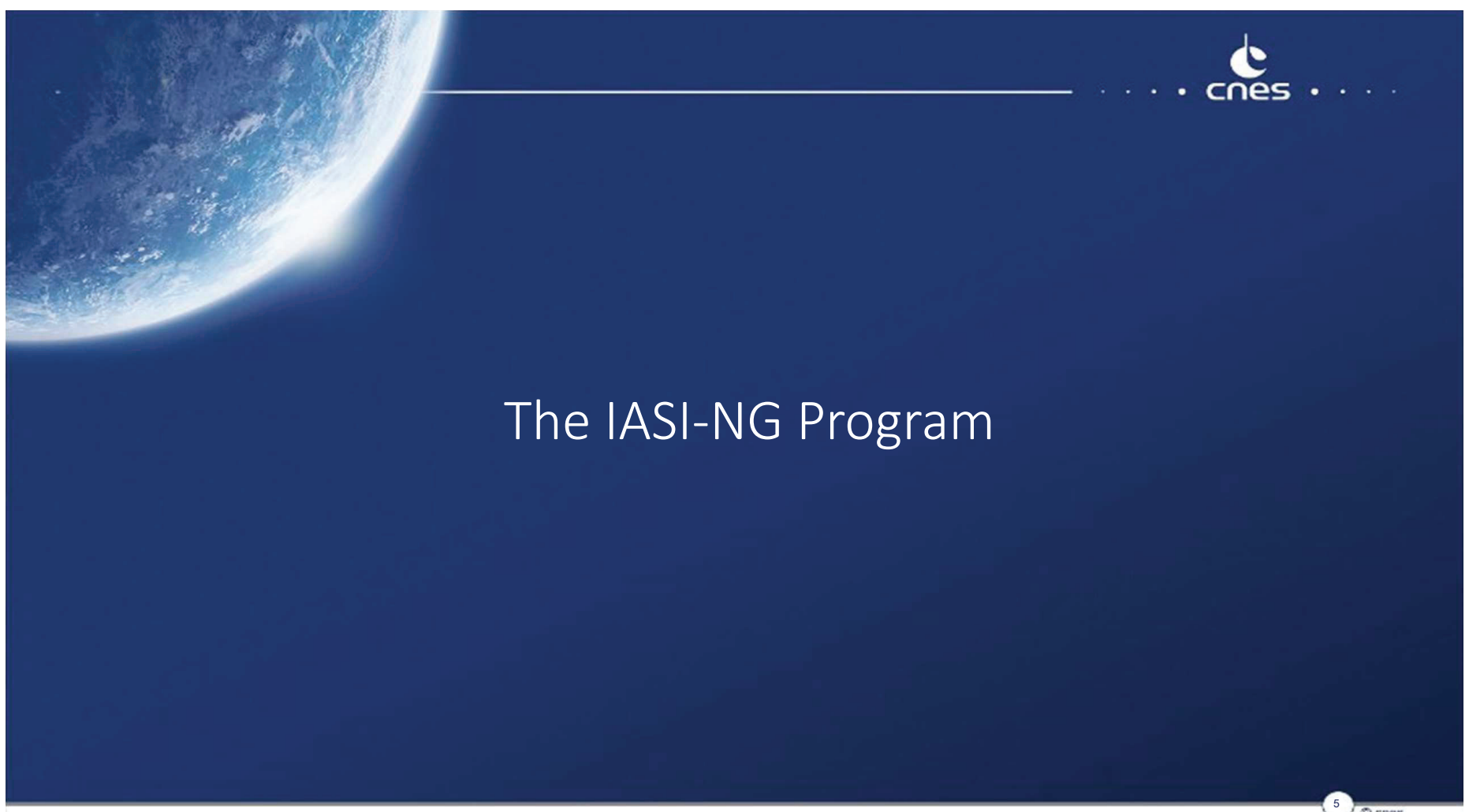

\section{IASI-NG and EPS-SG}

IASI-NG is part of the EUMETSAT EPS-SG program (European Polar Satellites). It will be on board Metop-SG A.

3 identical satellites will be launched in 2023, 2030 and 2037

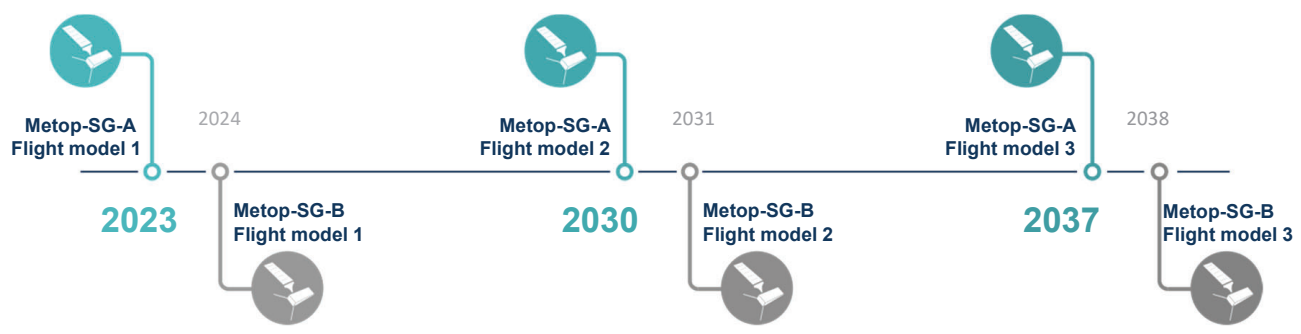

IASI-NG is an interferometer that will acquire spectra in the thermal infrared wavelength.

It is the successor of IASI ... well known for its positive impact on Weather Forecast and Atmospheric Composition studies 


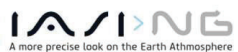

\section{| IASI-NG \\ An international Program}

\section{IASI-NG Development :}

- 3 instruments to be flown on the series Metop-SG A Satellites

- Level 1 Operational Processor (L1CPOP) processing scientific data up to level 1C

- Technical Expertise Center (IASTEC) performances monitoring in orbit

Partners

- Eumetsat : EPS-SG system development,

- ESA : Metop-SG A \& B Satellite development.

- Norway, UK, Swiss Space Agencies

Industry

Different instrument concepts trade-off performed : A Mertz interferometer allowing a field Effect compensation concept selected from Airbus DS proposal

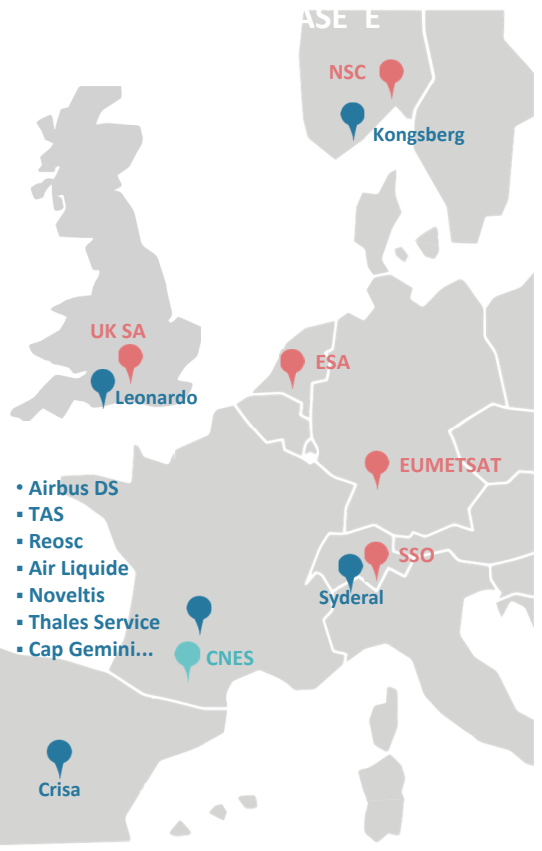

\section{Project organisation}

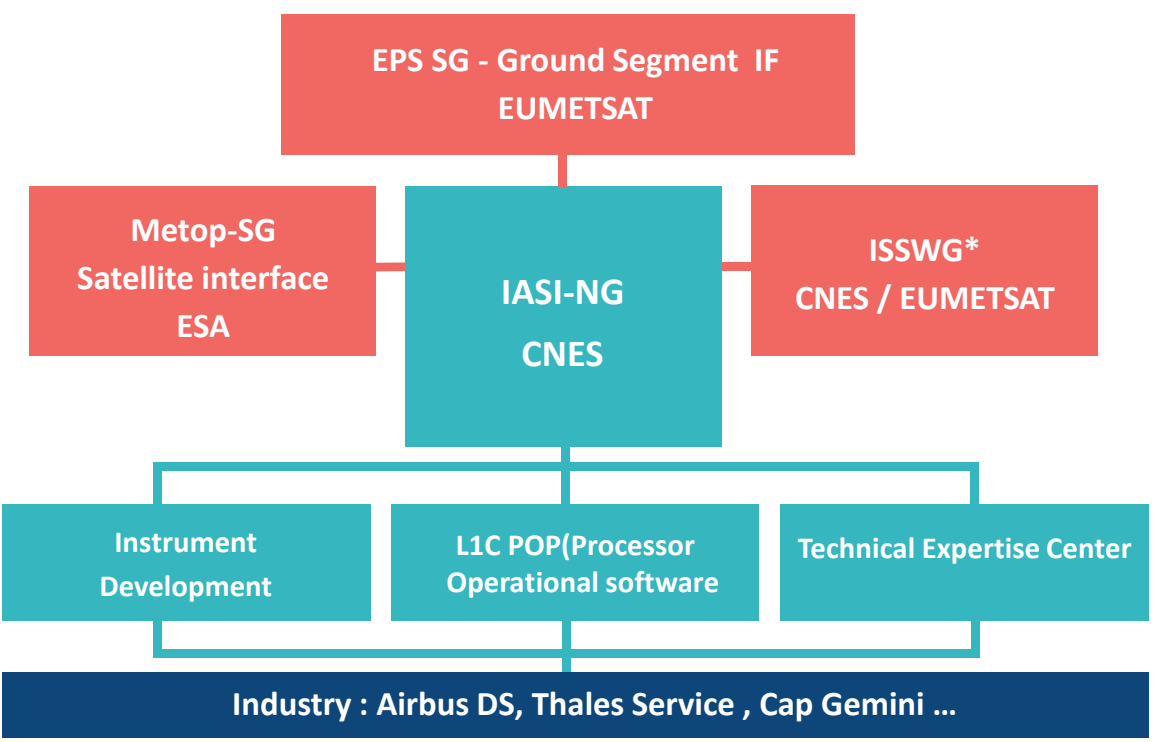


| L1C POP Development

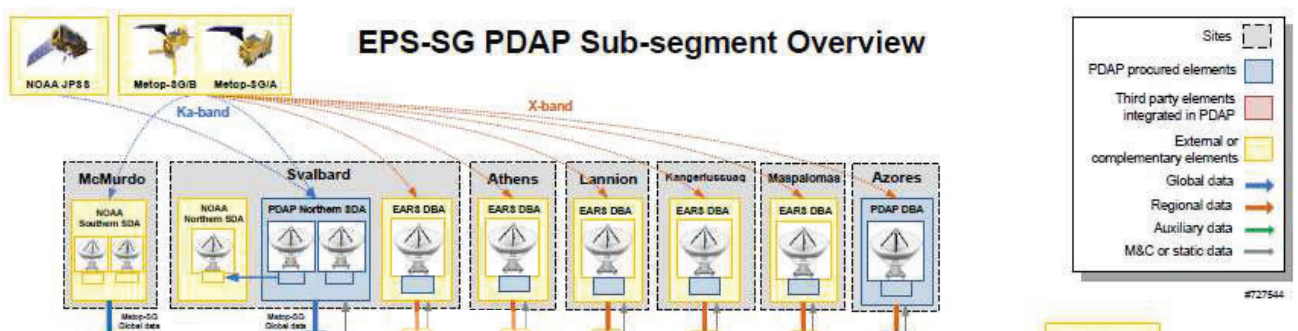

\section{IASI-NG L1C POP}

Integrated in Payload Data Acquisition and Processing component within EPS-SG overall ground segment at EUMETSAT

\section{DATA FLOW \\ MOVIE}




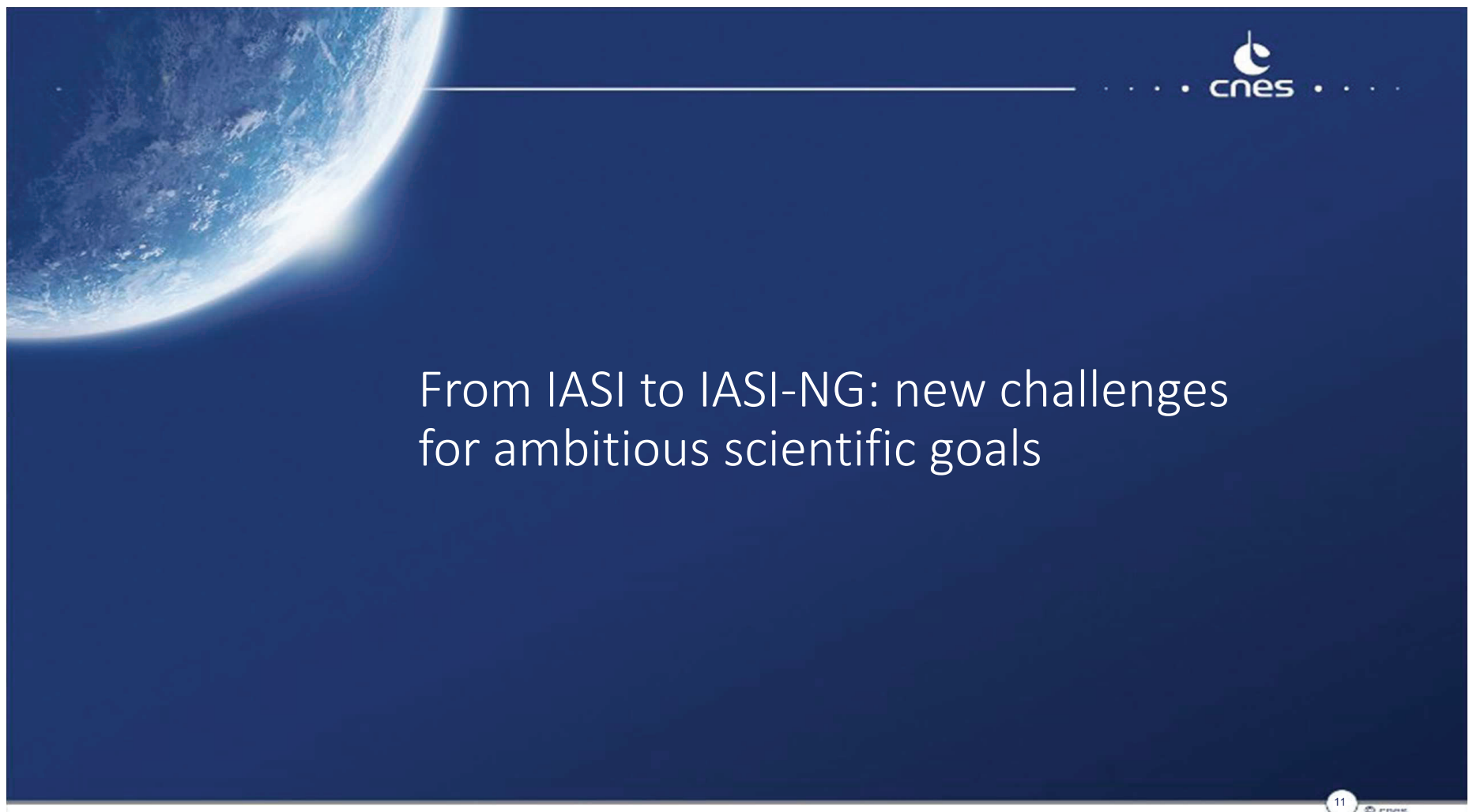

- Passive measurements of Earth-atmosphere radiations

- Detection and quantification of gases based on measurements of absorption features in the spectrum

- Retrieval of concentrations profiles thanks to the high spectral resolution
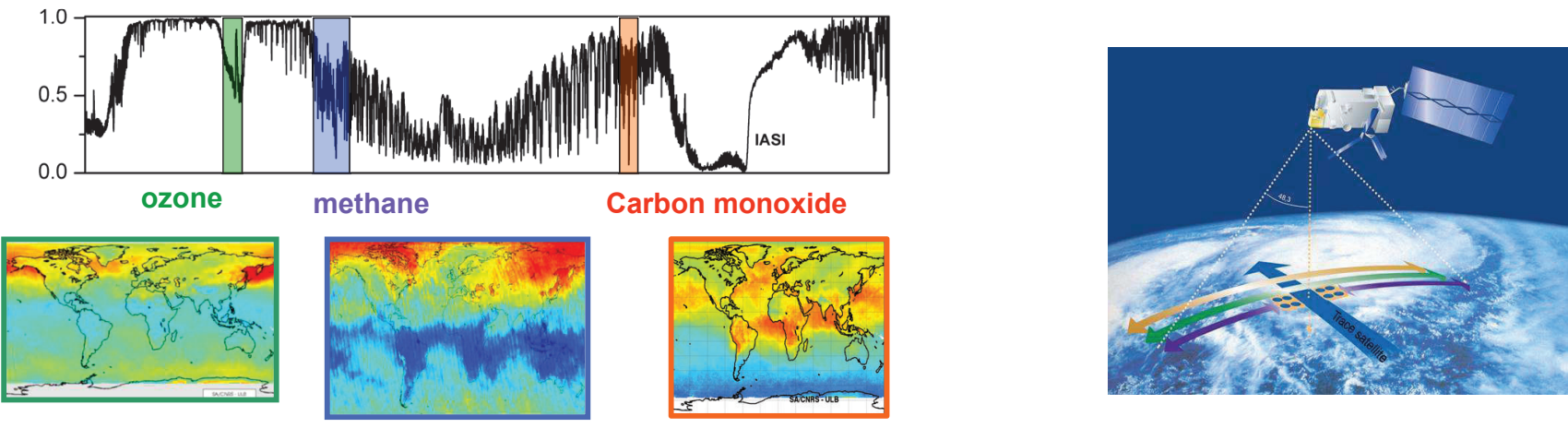

C. Clerbaux LATMOS/ULB 


\section{$1 \wedge<1>E$}

The targeted applications for IASI and IASI-NG are the same:

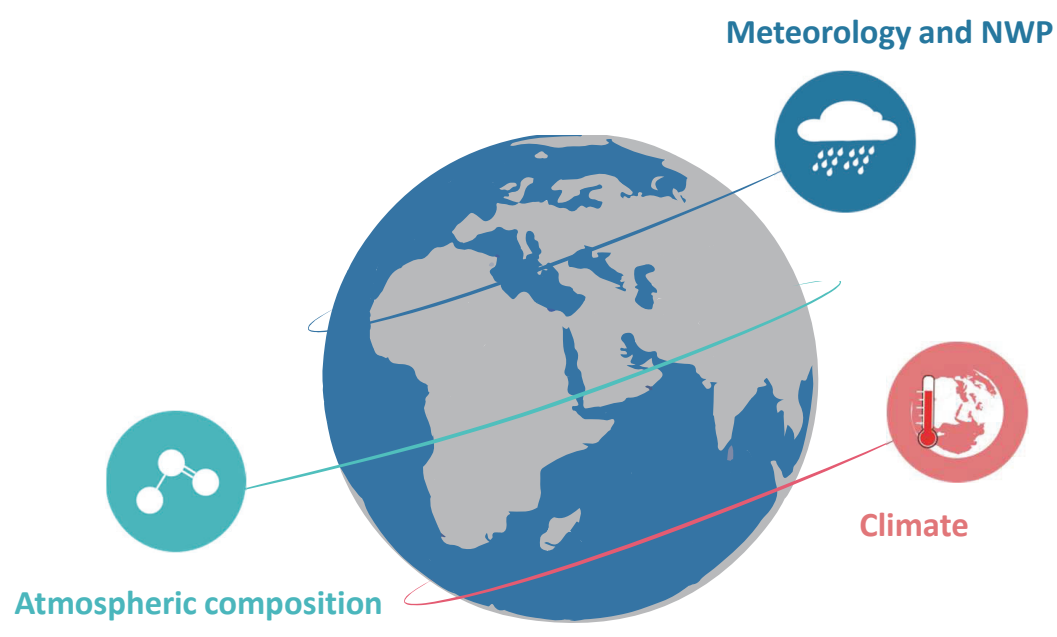

The breakthrough will be on the data quality (radiometric and spectral resolution)...

Several geophysical parameters may be retrieved from IASI spectra:

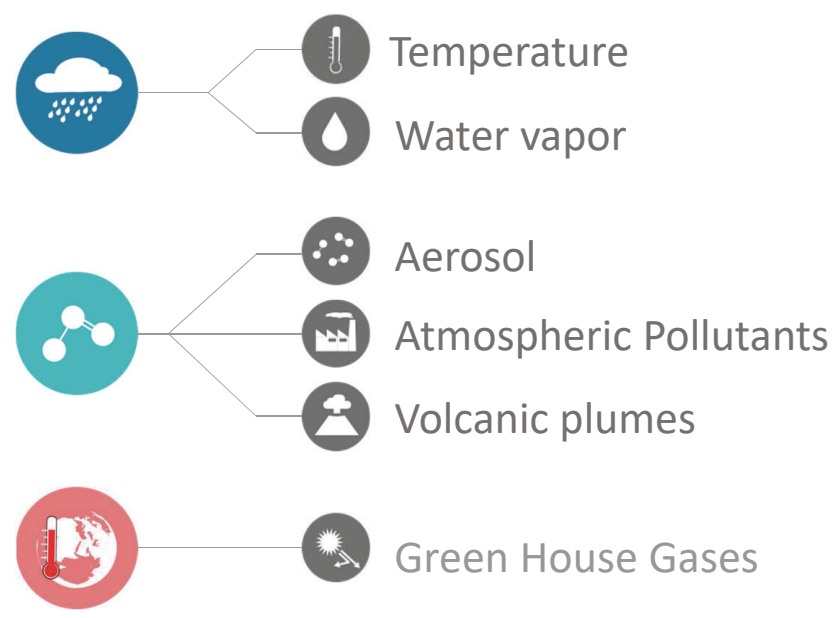




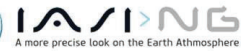

Importance of IASI data in the assimilation schemes for NWP

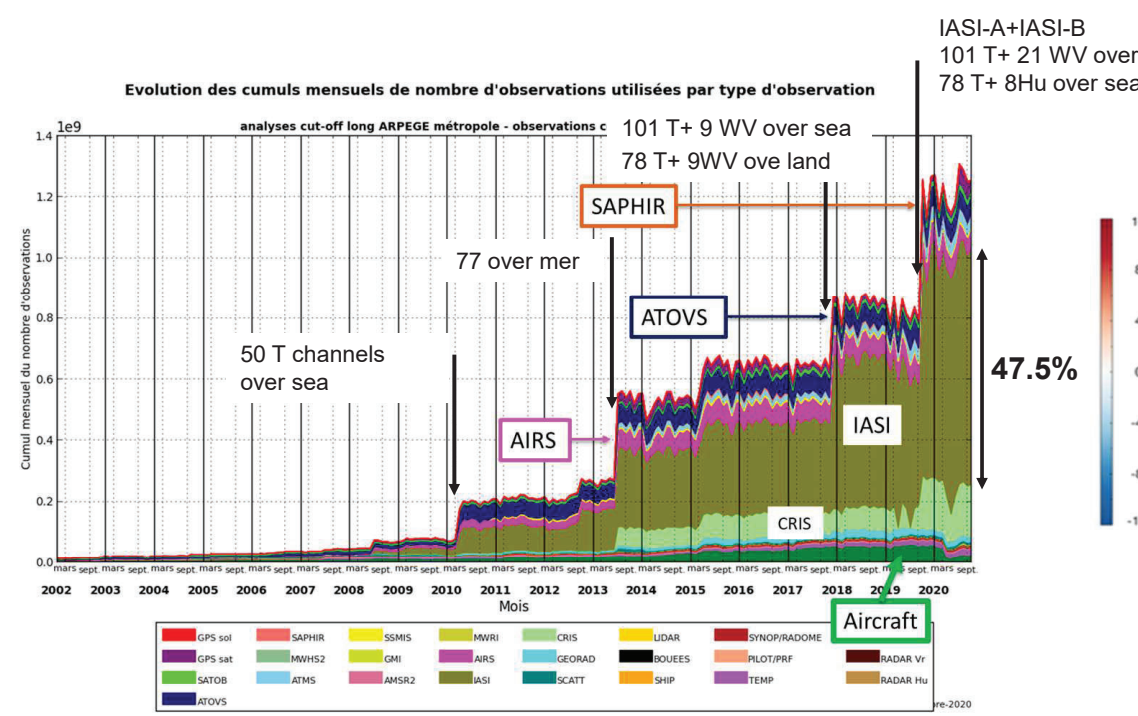

Météo-France

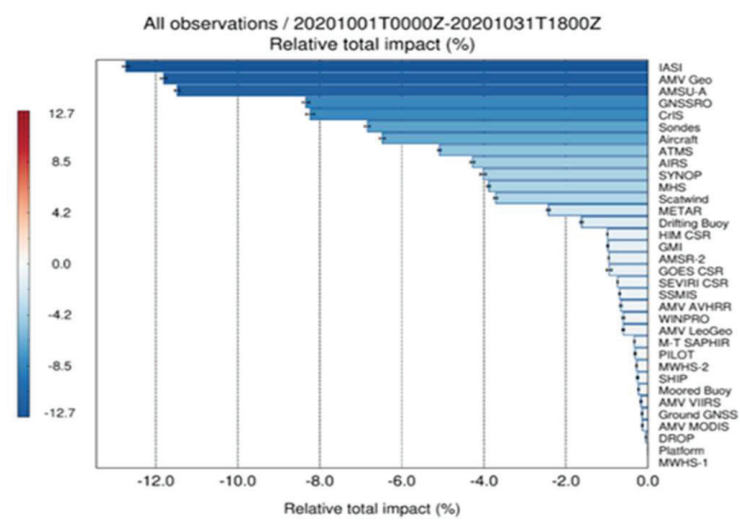

UK MetOffice

The new challenges of NWP .. for which IASI-NG should be helpful

\section{Surfaces}

Retrieved temperature in $\mathrm{K}$ (clear sky)

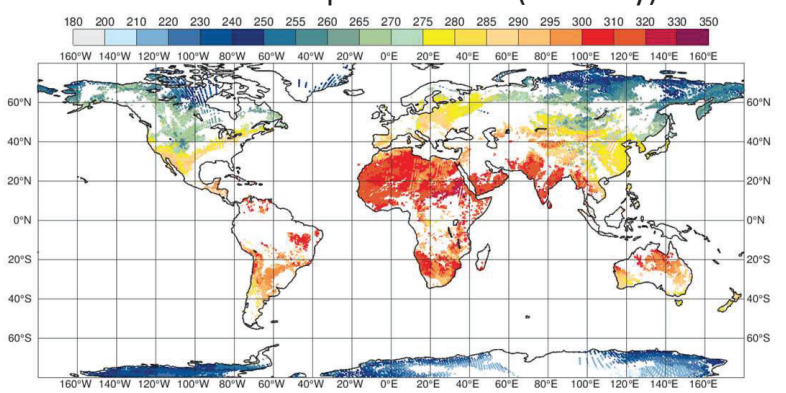

and

clouds

Pressure on top of the clouds ( $\mathrm{hPa})$

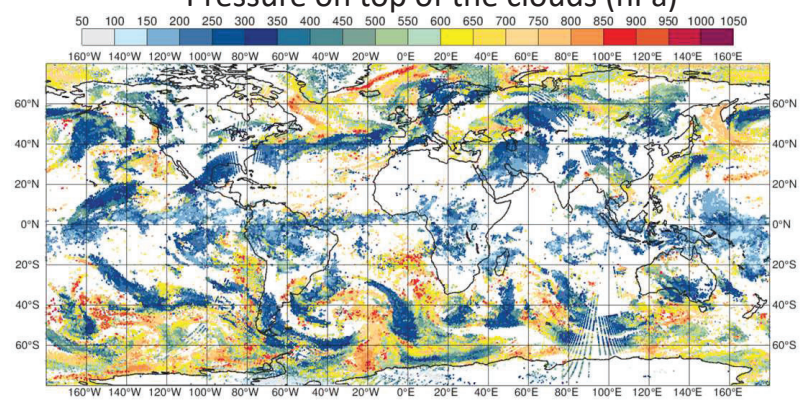

Chemistry: ozone, $\mathrm{CO}_{2}, \mathrm{CH}_{4}, \mathrm{CO}, \mathrm{N}_{2} \mathrm{O}$

Towards an integrated Earth system ...

=> Increasing number of IASI channels that are operationally assimilated 
ICSO 2020

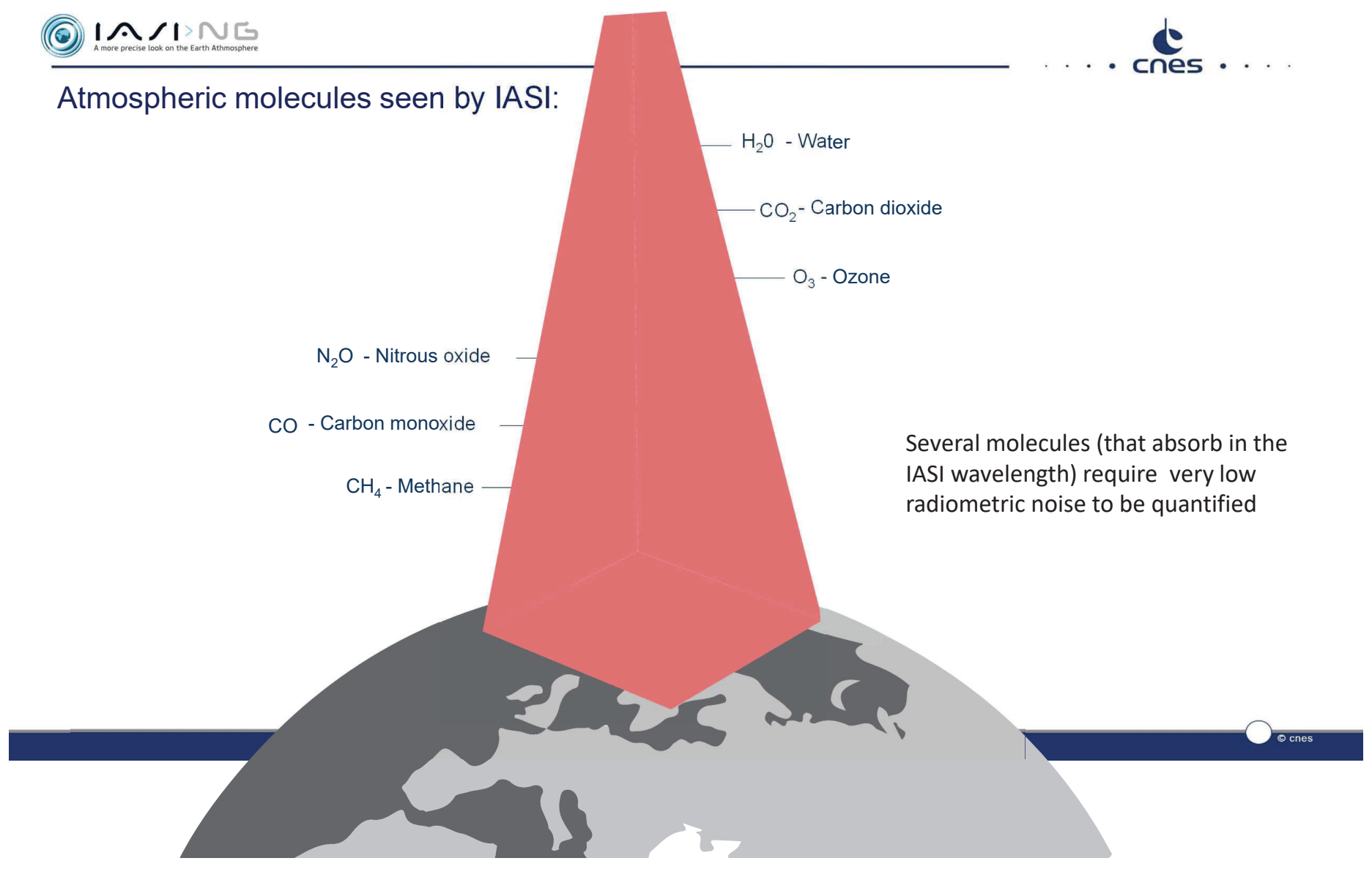

$1 \curvearrowright<1>0$

\section{Climate}

Most of atmospheric Essential Climate Variables (ECV) can be observed by IASI, day and night.

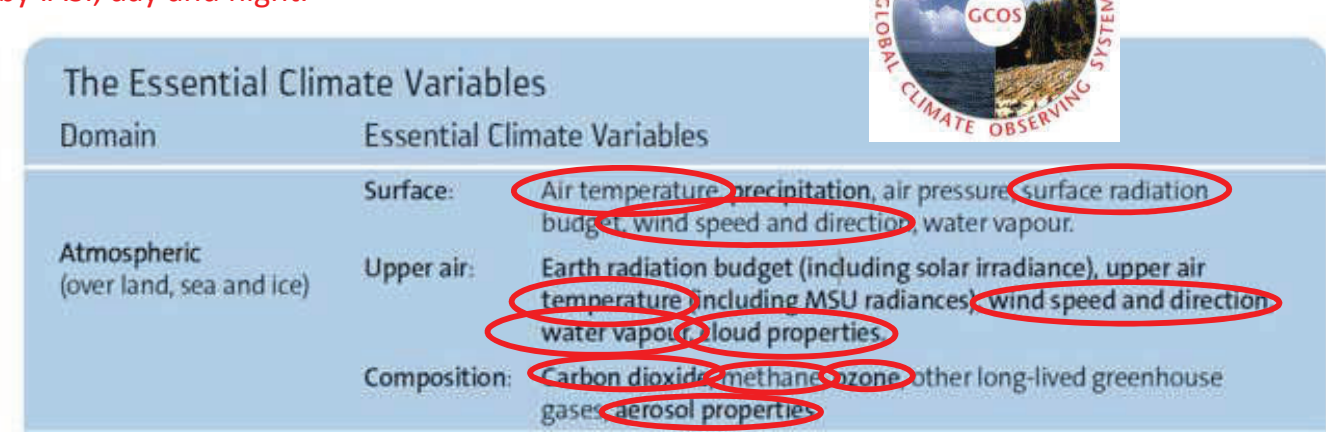

\section{7 atmospheric ECV}

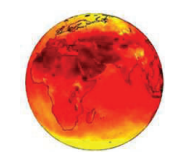

Temperature

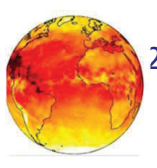

$\mathrm{H}_{2} \mathrm{O}$

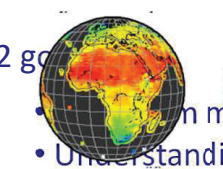
temperature

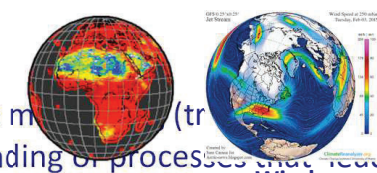

Emissivity

Wind

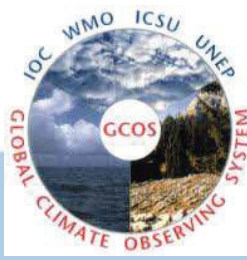

cres

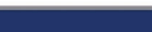

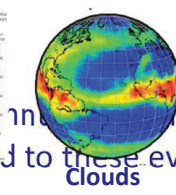

t

clouds

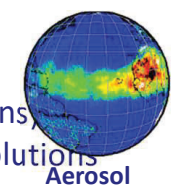

Aerosol
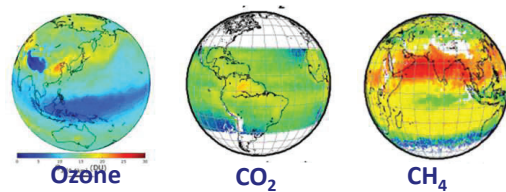

$\mathrm{CH}_{4}$ 


\section{I^ノ1 NE}

The trends

to be

observe are

very small
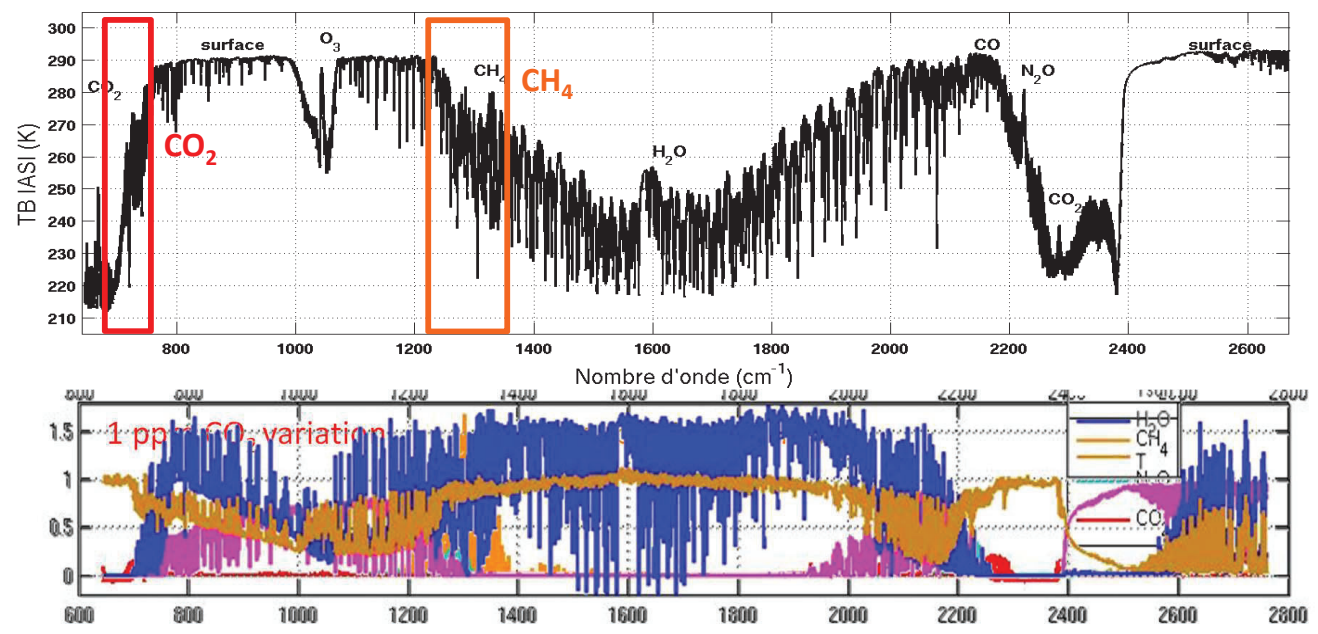

$\mathrm{H}_{2} \mathrm{O}$ Temp.

$1 \mathrm{ppm} \mathrm{CO} 2$ variation $=0,1 \mathrm{~K}$ !!

Trend : 2ppm.an ${ }^{-1}=0,2 \mathrm{~K} \cdot \mathrm{an}^{-1}$ !!

$\rightarrow$ A very accurate calibration of the instrument is needed

IASI-NG will acquire spectra in the same wavelength as IASI does

- With a two times better spectral resolution

- A two times lower radiometric noise

\section{Which gain for the scientific applications?}

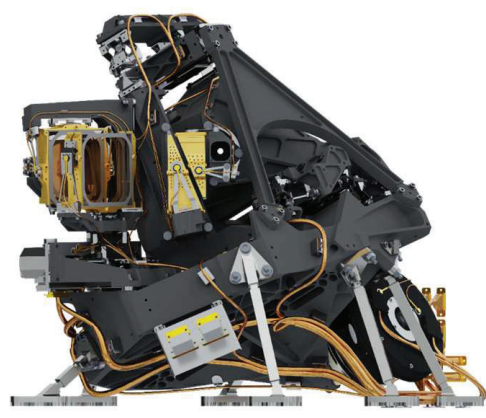

- Better estimation of atmospheric species (especially in the troposphere)

- Trace gases retrieval in an higher number of atmospheric levels 


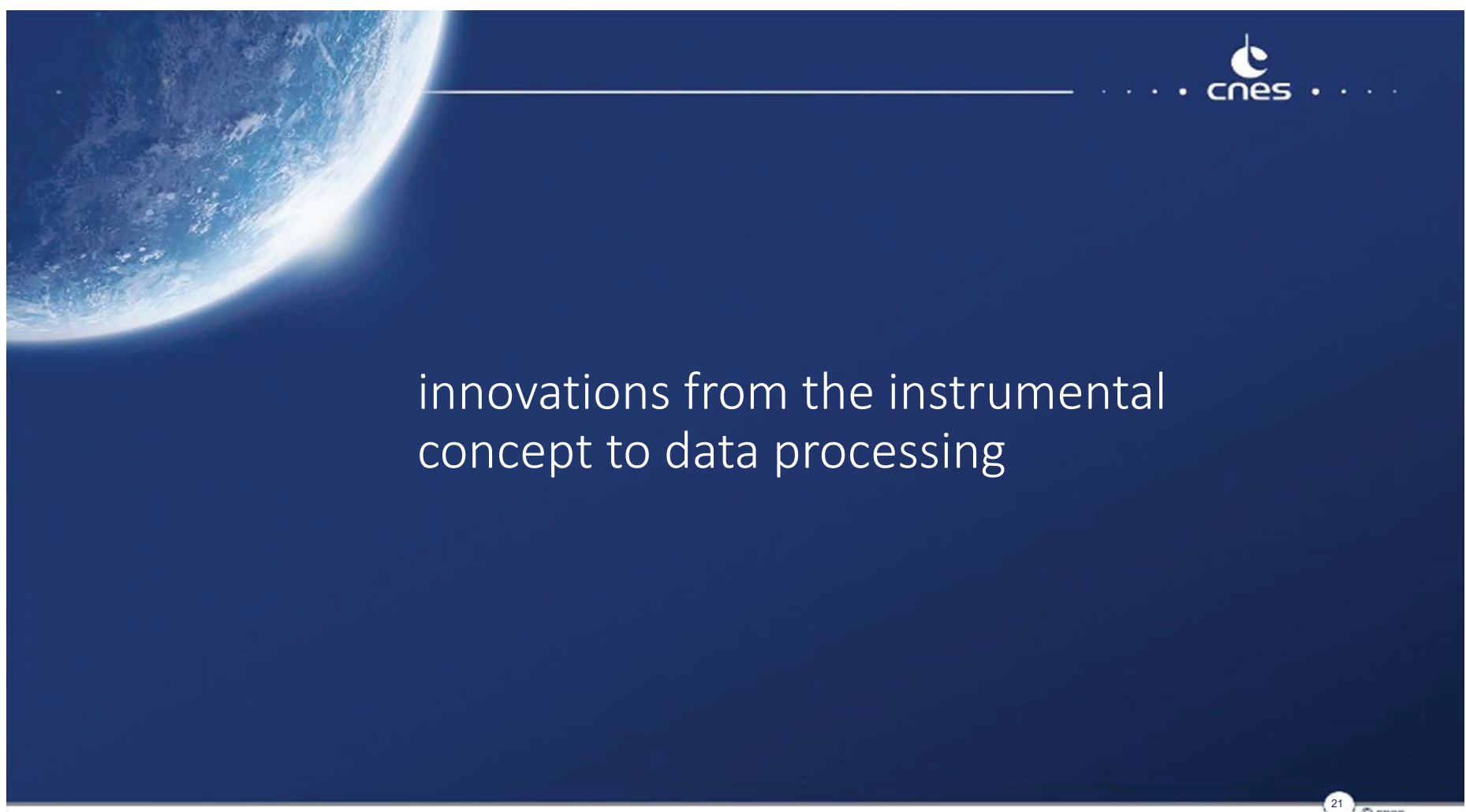

From IASI to IASI-NG: innovations and new challenges

\section{IASI-NG Level 1 main characteristics}

\begin{tabular}{|l|l|l|}
\hline Main figures & IASI & IASI-NG \\
\hline $\begin{array}{l}\text { Radiometric Resolution (NeDT) } \\
\text { Spectral resolution }\end{array}$ & $0.5 \mathrm{~cm}^{-1}$ & IASI/2 \\
\hline Absolute Radiometric Calibration & $<0,5 \mathrm{~K}$ & IASI/2 (0.25 $\mathrm{cm}^{-1} @$ LC) \\
\hline $\begin{array}{l}\text { Spectral bands } \\
\text { Number of sounder pixels per acquisition }\end{array}$ & 3 bands & IASI/2(<0,25K@280K) \\
\hline Ground Pixel diameter & 4 pixels & 4 bands \\
\hline Ground sampling & $12 \mathrm{~km}$ & 16 pixels \\
\hline
\end{tabular}

To meet these challenging requirements, innovations were needed...

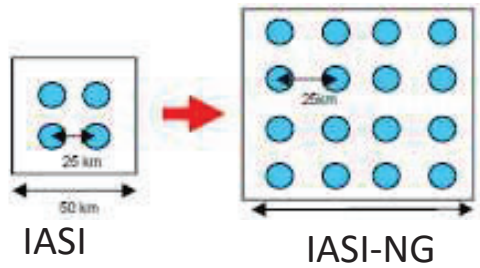




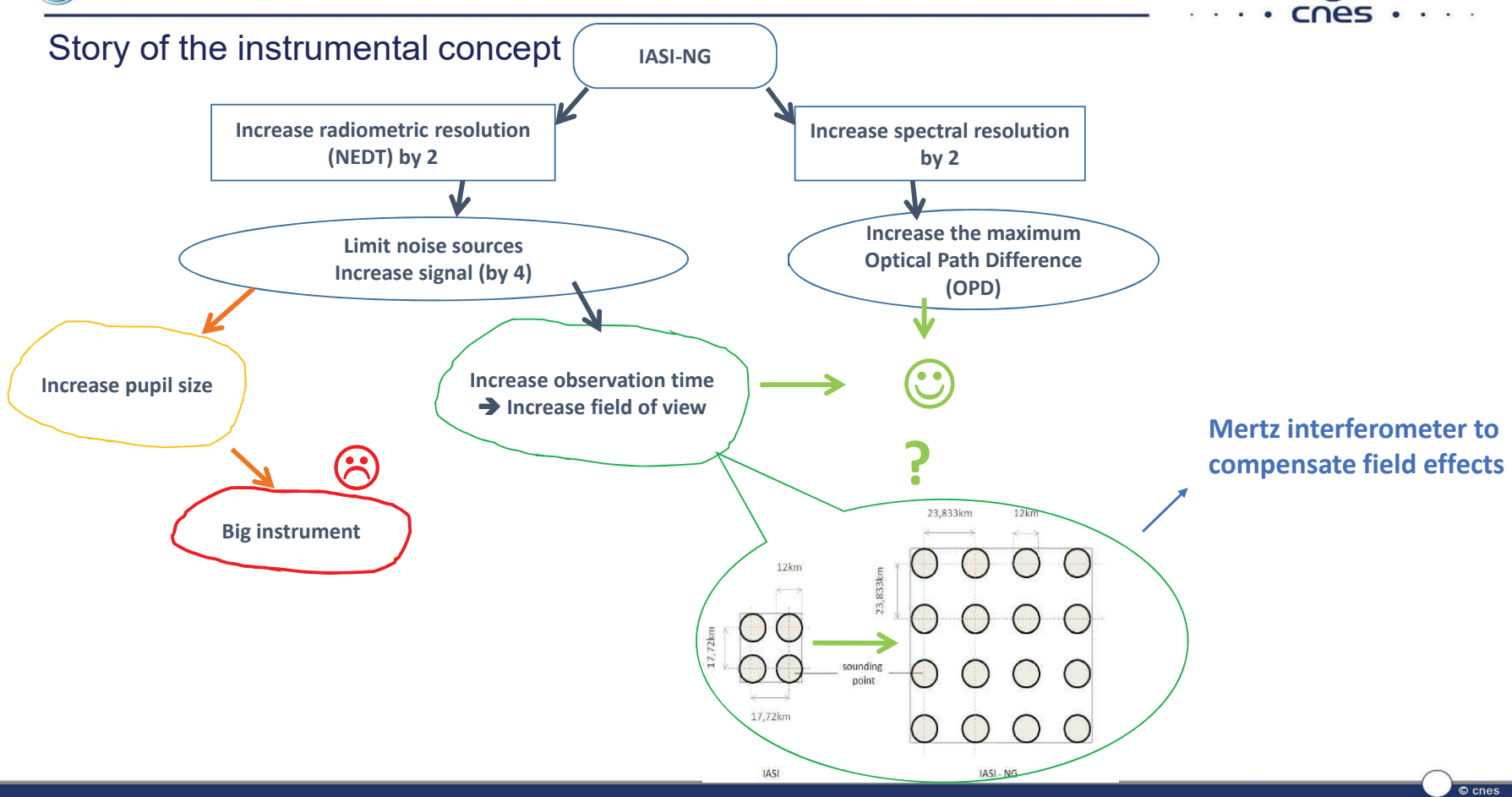

- IASI vs IASI-NG ?

\section{$\rightarrow \quad$ With great performance comes great complexity}

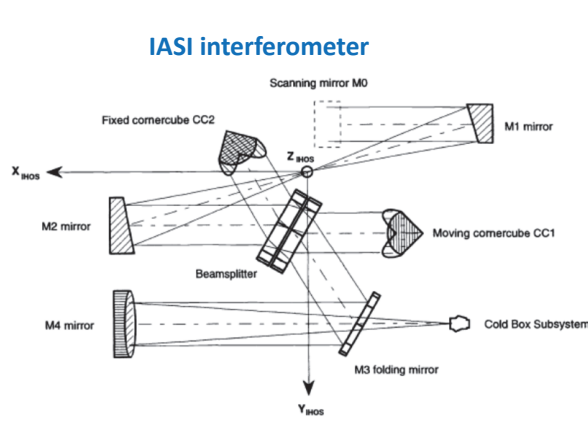

Figure 3.1-1 : IHOS optical configuration

- 4 optical components

- 1 moving corner cube

- nearly achromatic / all reflective design

- 1 laser trigs the acquisition

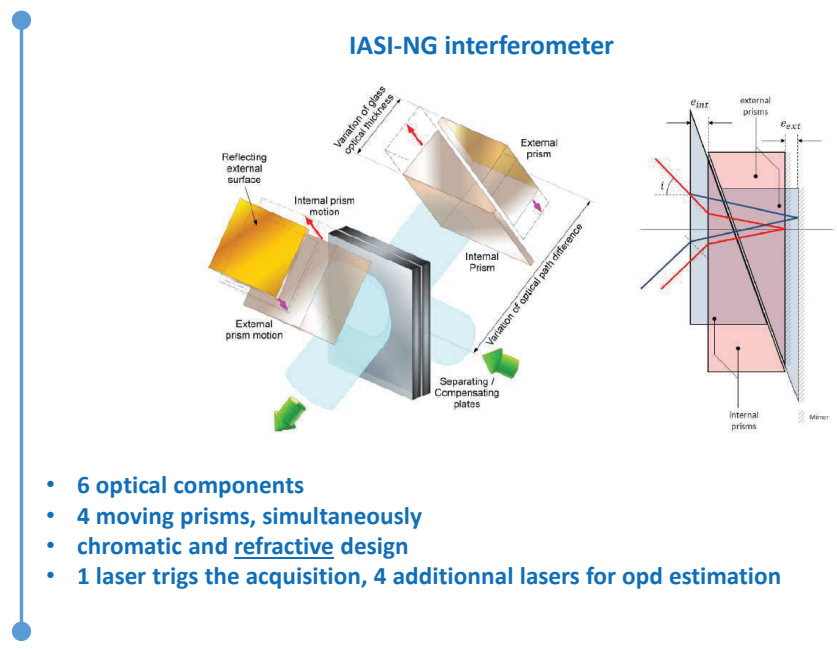




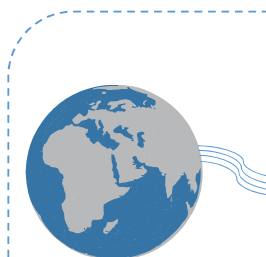

Earth radiation

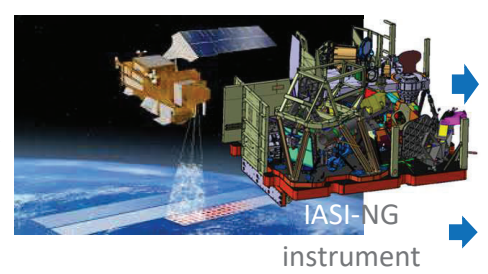

instrument

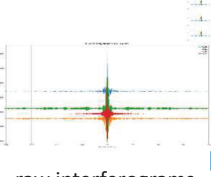

raw interferograms

(4 bands, 16 FOV)

\section{.}

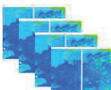

filtered images

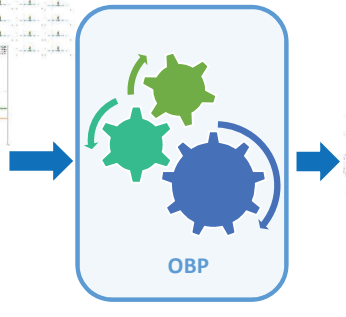

$\vec{r}$

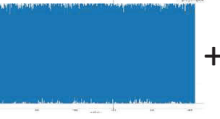

LO complex spectra

(compressed)

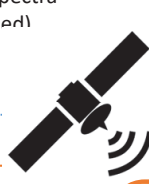

on-ground processing
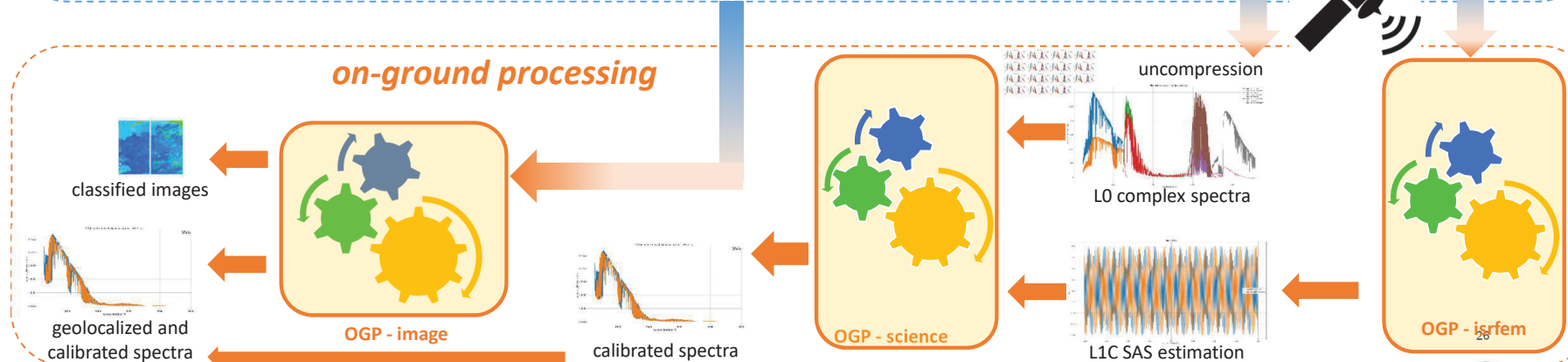

ocnes 
- ISRF estimation: the second main innovation

$>$ ISRF is estimated for every set of $16 \times 4$ acquisitions

$$
\text { estimating the ISRF = knowing the science opd }
$$

Airbus D\&S shows that the opd for every wavelength in the science band can be approximated by a linear combination of the 5 metrologies opd :

$$
\operatorname{opd}(\sigma)=\left(\mathbf{A}_{\text {cal }}(\sigma)-\mathbf{C}_{0}\left(\sigma, \mathbf{Z}_{0}\right)\right) \cdot \mathbf{Z}_{0}+\sum_{\mathrm{i}=1}^{4} \mathrm{C}_{\mathbf{i}}\left(\sigma, \mathbf{Z}_{0}\right) \cdot \mathbf{Z}_{\mathbf{i}}+\operatorname{OFFSET}\left(\sigma, \mathbf{Z}_{0}\right)
$$

$\mathbf{Z}_{\mathbf{i}=\mathbf{1 . 4}} \quad$ : metrologies opd

$\mathbf{Z}_{\mathbf{0}} \quad$ : reference metrology opd

$\mathbf{C}_{\mathbf{i}=\mathbf{0 . . 5}}$ : opd coefficients 


\section{- IASI-NG ISRF estimation principle}

$>$ ISRF-Estimation Model parameters - Coefficients

$$
\operatorname{opd}(\sigma)=\left(\mathbf{A}_{\text {cal }}(\sigma)-\mathrm{C}_{0}\left(\sigma, \mathbf{Z}_{0}\right)\right) \cdot \mathbf{Z}_{0}+\sum_{\mathrm{i}=1}^{4} \mathrm{C}_{\mathrm{i}}\left(\sigma, \mathrm{Z}_{0}\right) \cdot \mathrm{Z}_{\mathrm{i}}+\operatorname{OFFSET}\left(\sigma, \mathbf{Z}_{0}\right)
$$

$\mathbf{Z}_{\mathbf{i}=\mathbf{0 . . 4}}:$ metrologies opd

$\mathrm{Z}_{5}=1$

$\mathbf{C}_{\mathbf{i}=\mathbf{0 . . 5}}$ : opd coefficients

They are a combination of :

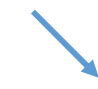

\section{- IASI-NG ISRF estimation principle}

$\mathbf{Z}_{\mathbf{i}=\mathbf{0 . . 4}}:$ metrologies opd

$>$ ISRF-Estimation Model parameters - Coefficients

$\mathrm{Z}_{5}=\mathbf{1}$

$\mathbf{C}_{\mathbf{i}=\mathbf{0 . 5}}$ : opd coefficients

$$
\operatorname{opd}(\sigma)=\left(\mathrm{A}_{\text {cal }}(\sigma)-\mathrm{C}_{0}\left(\sigma, \mathbf{Z}_{0}\right)\right) \cdot \mathbf{Z}_{0}+\sum_{\mathrm{i}=1}^{4} \mathrm{C}_{\mathrm{i}}\left(\sigma, \mathbf{Z}_{0}\right) \cdot \mathbf{Z}_{\mathrm{i}}+\operatorname{OFFSET}\left(\sigma, \mathbf{Z}_{0}\right)
$$

Measured coefficient

They are a combination of

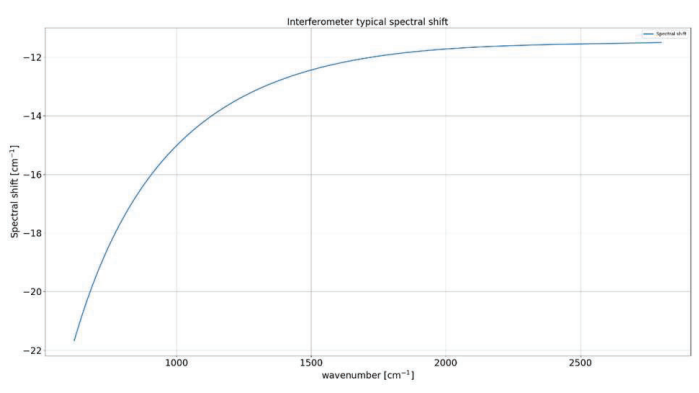




\section{- IASI-NG ISRF estimation principle}

$>$ ISRF-Estimation Model parameters - Coefficients - $A_{\text {cal }}$

The $\mathrm{A}_{\text {cal }}$ coefficient is related to the spectral shift $\Delta \boldsymbol{\sigma}$ throught the simple relation :

$$
\mathrm{A}_{\text {cal }}(\sigma)=\frac{\Delta \sigma}{\sigma}
$$

1- $\mathbf{A}_{\text {cal }}(\boldsymbol{\sigma})$ values are computed for few wavenumbers (anchor points) across the IASI-NG band using correlation between observed and modeled spectra.

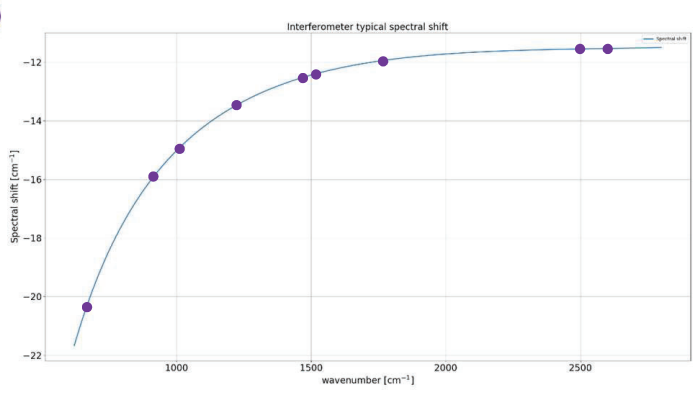

\section{- IASI-NG ISRF estimation principle}

$>$ ISRF-Estimation Model parameters - Coefficients - $A_{\text {cal }}$

The $A_{\text {cal }}$ coefficient is related to the spectral shift $\Delta \boldsymbol{\sigma}$ throught the simple relation :
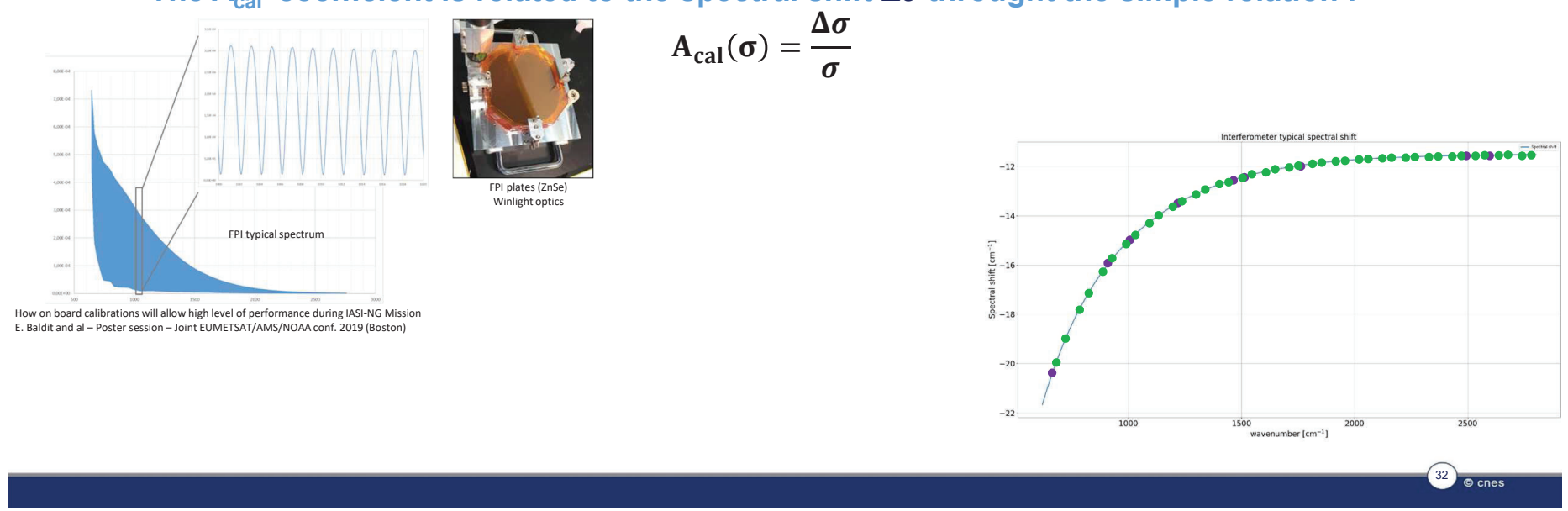


\section{|ASI-NG Instrument Flight Model Integration and Test}
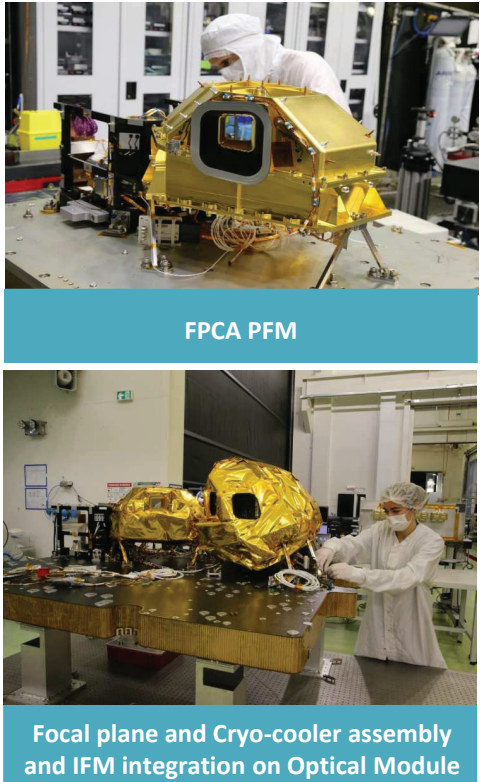
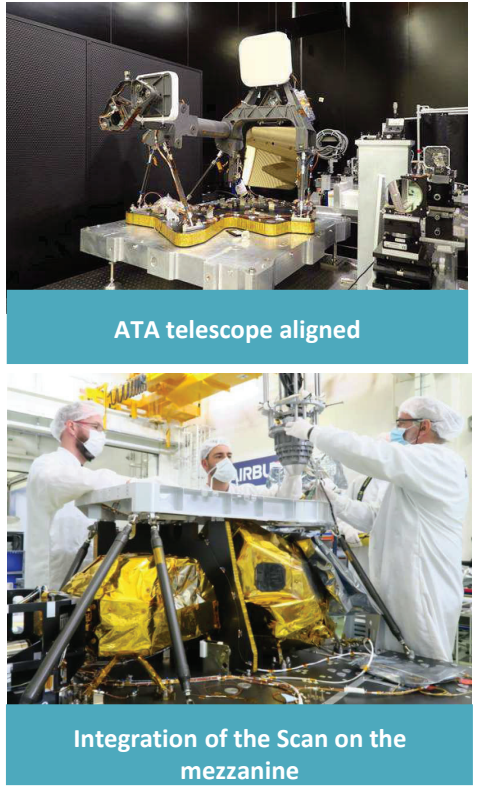
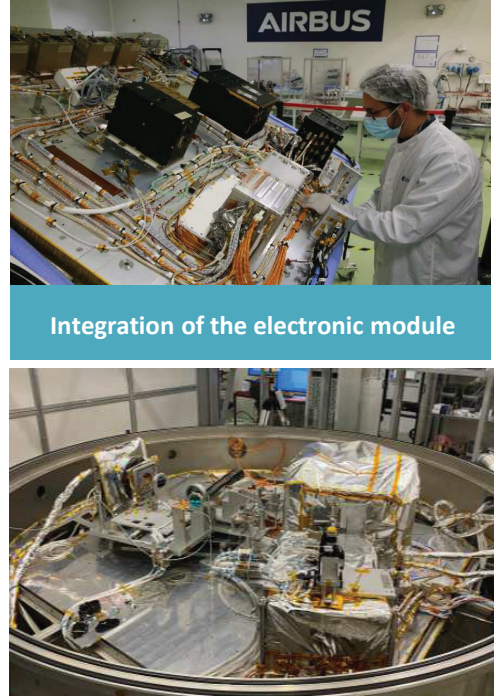

vacuum performance test preparation with the PFM interferometer

- Synthesis of the current performances budget (compared to specifications at mission level) for the main requirements

\begin{tabular}{|c|c|c|c|c|}
\hline $\begin{array}{l}\text { Mission } \\
\text { Measurement } \\
\text { Requirement }\end{array}$ & $\begin{array}{l}\text { Corresponding System } \\
\text { Requirement }\end{array}$ & Description & $\begin{array}{l}\text { Specified value } \\
\text { / calculated } \\
\text { value }\end{array}$ & Status \\
\hline SRD.IAS_20 & $\begin{array}{l}\text { SYS_ISRFM_070_SPACE } \\
\text { SYS_ISRFM_080_SYS }\end{array}$ & $\begin{array}{l}\text { Interpixel } \\
\text { ISRF stability } \\
\text { ISRF shift } \\
\text { knowledge }\end{array}$ & $\begin{array}{l}\text { 1E10-4 / 3.3E10- } \\
5 \\
1 \text { E10-6/ } \\
1.145 E 10-6 \text { (WC) }\end{array}$ & $\begin{array}{l}\text { compliant } \\
\text { (partially) }\end{array}$ \\
\hline$\overline{\text { SRD.IAS_60 }}$ & $\begin{array}{l}\text { SYS_ISRFM_080_SYS } \\
\text { SYS_ISRFM_130_SYS }\end{array}$ & $\begin{array}{l}\text { ISRF shift } \\
\text { knowledge } \\
\text { Shape error }\end{array}$ & $\begin{array}{l}1 \text { 1E10-6 / } \\
1.145 E 10-6 \text { (WC) } \\
\text { See Figure } 2\end{array}$ & $\begin{array}{l}\text { Marginally } \\
\text { NC }\end{array}$ \\
\hline$\overline{\text { SRD_IAS.90 }}$ & $\begin{array}{l}\text { SYS_PIPR_020_SPACE } \\
\text { SYS_PIPR_065_GRND }\end{array}$ & $\begin{array}{l}\text { Radiometric } \\
\text { noise }\end{array}$ & See Figure 5 & $\begin{array}{l}\text { Marginally } \\
\text { NC }\end{array}$ \\
\hline SRD_IAS.100 & SYS_PIPR_075_SYS & $\begin{array}{l}\text { Absolute } \\
\text { radiometric } \\
\text { calibration }\end{array}$ & $\begin{array}{l}0.25 \mathrm{~K}(\mathrm{O}) \text { and } \\
0.5 \mathrm{~K}(\mathrm{~T}) / 0.24 \mathrm{~K}\end{array}$ & compliant \\
\hline SRD_IAS.110 & SYS_PIPR_100_SPACE & $\begin{array}{l}\text { Radiometric } \\
\text { bias }\end{array}$ & $\begin{array}{l}0.1 \mathrm{~K}(\mathrm{O}) \text { and } \\
0.05 \mathrm{~K}(\mathrm{~T}) / \\
0.125 \mathrm{~K}\end{array}$ & $\begin{array}{l}\text { Non } \\
\text { compliant }\end{array}$ \\
\hline SRD_IAS.120 & SYS_PIPR_110_SPACE & $\begin{array}{l}\text { Lifetime } \\
\text { radiometric } \\
\text { stability }\end{array}$ & $0.1 \mathrm{~K} / 0.42 \mathrm{~K}$ & $\begin{array}{l}\text { Non } \\
\text { compliant }\end{array}$ \\
\hline SRD_IAS.130 & SYS_PIPR_120_SPACE & $\begin{array}{l}\text { Interchannel } \\
\text { radiometric } \\
\text { bias }\end{array}$ & $0.1 \mathrm{~K} / 0.084 \mathrm{~K}$ & compliant \\
\hline SRD_IAS.140 & SYS_PIPR_130_SPACE & $\begin{array}{l}\text { Intersscan/int } \\
\text { erpixel } \\
\text { radiometric } \\
\text { bias }\end{array}$ & $0.1 \mathrm{~K} / 0.07 \mathrm{~K}$ & compliant \\
\hline
\end{tabular}

\begin{tabular}{|c|c|c|c|c|}
\hline $\begin{array}{l}\text { Mission } \\
\text { Measurement } \\
\text { Requirement }\end{array}$ & $\begin{array}{l}\text { Corresponding System } \\
\text { Requirement }\end{array}$ & Description & $\begin{array}{l}\text { Specified value / } \\
\text { calculated value }\end{array}$ & Status \\
\hline SRD_IAS.150 & SYS_GEOM_010_SPACE & Spatial coverage & By design & compliant \\
\hline SRD_IAS.160 & SYS_GEOM_090_SPACE & Spatial resolution & $\begin{array}{l}11.5+/-0.5 \mathrm{~km} / \\
11.8 \mathrm{~km}\end{array}$ & compliant \\
\hline SRD_IAS.170 & SYS_GEOM_100_SPACE & Off nadir resolution & - & compliant \\
\hline SRD.IAS_180 & SYS_GEOM_040_SPACE & Pointing stability & $\begin{array}{c}0.1 \mathrm{mrad} / 0.062 \\
\mathrm{mrad}\end{array}$ & compliant \\
\hline \multirow[t]{2}{*}{$\begin{array}{l}\text { SRD_IAS.190 } \\
\text { SRD_IAS.195 }\end{array}$} & $\begin{array}{l}\text { SYS_FMISSION_040_SYS } \\
\text { SYS_FMISSION_045_SYS }\end{array}$ & $\begin{array}{l}\text { Sounder } \\
\text { geolocation }\end{array}$ & $\begin{array}{l}1-5 \mathrm{~km} / 601-950 \mathrm{~m} \\
1.745 \mathrm{mrad} / 1.188 \mathrm{mr} \\
\text { ad }\end{array}$ & compliant \\
\hline & SYS_LV1_110_GRND & $\begin{array}{l}\text { Geolocation in } \\
\text { degraded case }\end{array}$ & $5 \mathrm{~km} / 916 \mathrm{~m}$ & compliant \\
\hline SRD_IAS.200 & SYS_GEOM_070_SPACE & Pointing knowledge & $\begin{array}{c}+/-3 \mathrm{mrad} / \\
0.9 \mathrm{mrad}\end{array}$ & compliant \\
\hline SRD_IAS.220 & SYS_GEOM_020_SYS & Angular distance & $\begin{array}{c}{[25-50 \mathrm{mrad}] / /} \\
{[26.74-43.26 \mathrm{mard}]}\end{array}$ & compliant \\
\hline SRD_IAS.230 & SYS_GEOM_100_SPACE & Off nadir sampling & - & compliant \\
\hline SRD_IAS.235 & SYS_GEOM_030_SPACE & Pointing accuracy & $\begin{array}{l}+/-3 \mathrm{mrad} / \\
0.64 \mathrm{mrad}\end{array}$ & compliant \\
\hline SRD_IAS.250 & SYS_PIPSF_040_SPACE & $\begin{array}{l}\text { PSF uniformity } \\
\text { knowledge }\end{array}$ & $\begin{array}{l}+/-10 \% / 8 \% \text { in } \\
\text { typical case }\end{array}$ & compliant \\
\hline
\end{tabular}




\section{Other IASI-NG presentations in this conference}

Session 38 Spectrometer 7 - on Thursday, at 16:00 (France time):

- Talk 221. IASI-NG instrument development and performance status Elisa Baldit, CNES

- Talk 328. IASI NG Flight model development status Bertrand Calvel, Airbus Defense and Space

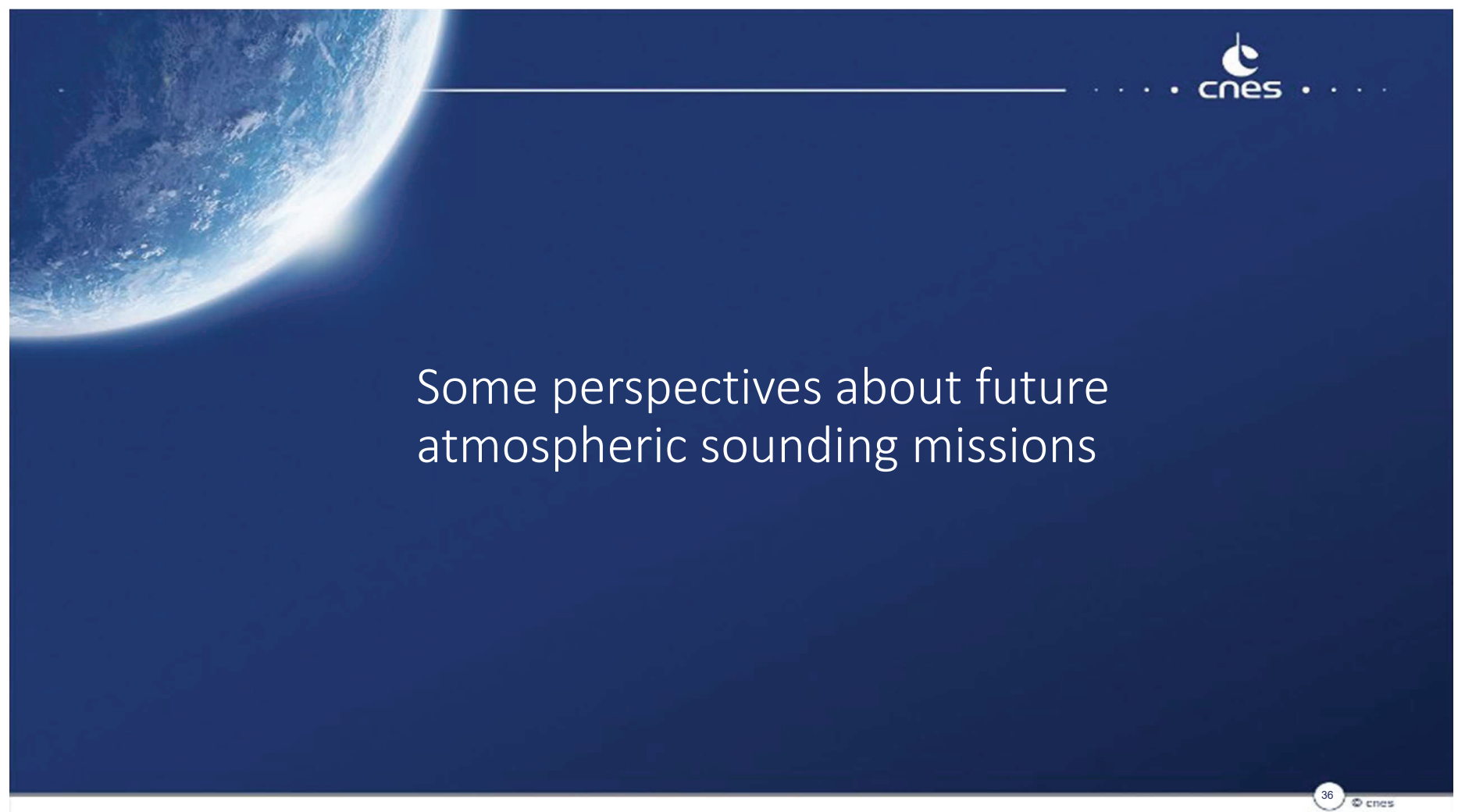




\section{CNES is supporting the ESA and EUMETSAT programs}

The future IR sounding in Europe is mainly ensured by the EPS-SG and MSG (Meteosat Third Generation) programs

+ FORUM mission (Earth Explorer 9) in Far Infrared

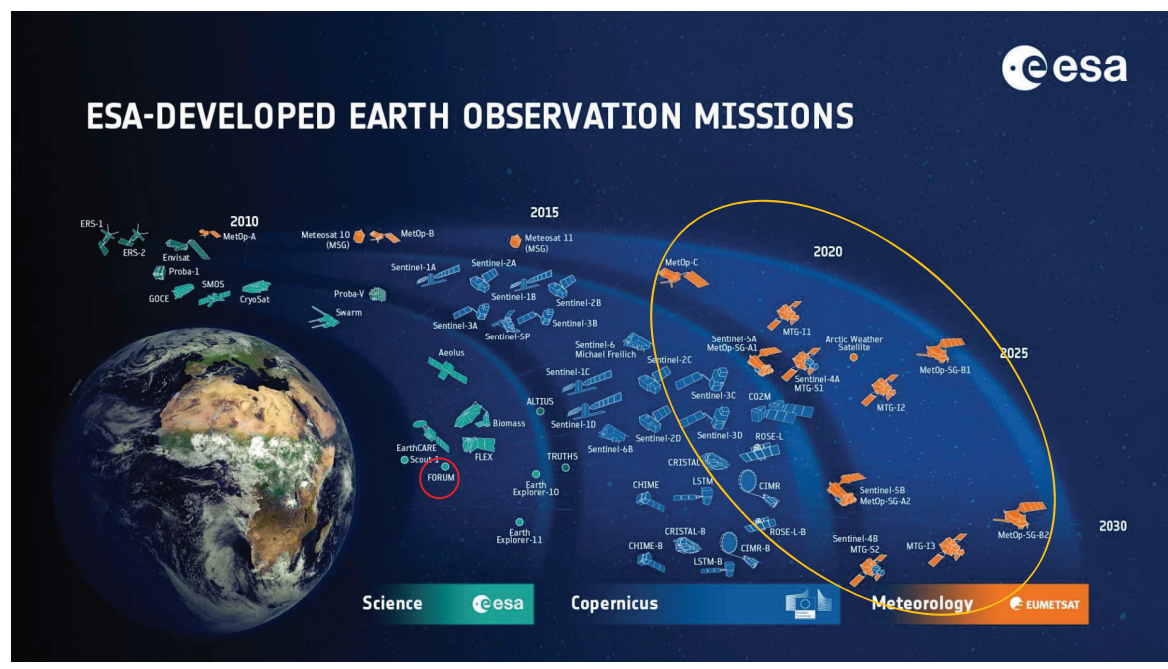

In addition of these ambitious programs, CNES is focusing on miniaturized instrument concepts

The need of meteorological users if to increase revisit

$\rightarrow$ constellation of small satellites can be a solution

The miniaturization of IR interferometers is a challenge...

Different R\&D and pre-development studies are on-going at CNES 
On-going studies on IR sounding missions aim at answering two different questions:

- How can we miniaturize interferometers without degrading the data quality?

$\rightarrow$ partial interferograms?

- What kind of constellation (orbit/ number of satellites) can provide data where "classic" data are missing the most?

$\rightarrow$ Experiment with Meteo-France (CMIM currently in phase 0 )

\section{Phases 0 and $A$ in Earth Observation at CNES}

- Phases A :

$\square$ WISA : wide-swath altimetry concept for Sentinel-3NG

SKIM : Ocean currents

ACCP (with NASA, C2OMODO/Tandem of MW radiometers)

- C 3 IEL (Study of cloud in 4D at decametric resolution, with ISA)

- SMASH (constellation of low-cost, miniaturized altimeters for operational hydrology)

- Transitioning to phase A :

$\square$ Nanomagsat (magnetic fields measured from nanosats)

$\square$ Hyperspectral

- SMOS-HR revisité ->SMOS Next phase A1 -(Soil Moisture and Ocean Salinity at higher spatial resolution)
Phase 0

- MARVEL : Mass And Reference Variations for Earth Lookout

- CARIOQA - continuation of GRICE

- ARGC : «Architecture Réactive pour la Gestion des Catastrophes »

- MALBEC : Meteor Automated Light Ballon Experimental Camera

E EXPE-VAL (lidar/hyperspectral - forets)

- CHIMERE (hyperspectral, coastal,vegetation)

SCALE (new lidar concept)

- CMIM - Constellation of Miniaturized IR sounders for Meteorology

- TIR on HAPS

IGC- TIR miniaturized imager for Aérosols, T and $\mathrm{H} 2 \mathrm{O}$ (with TAS) 


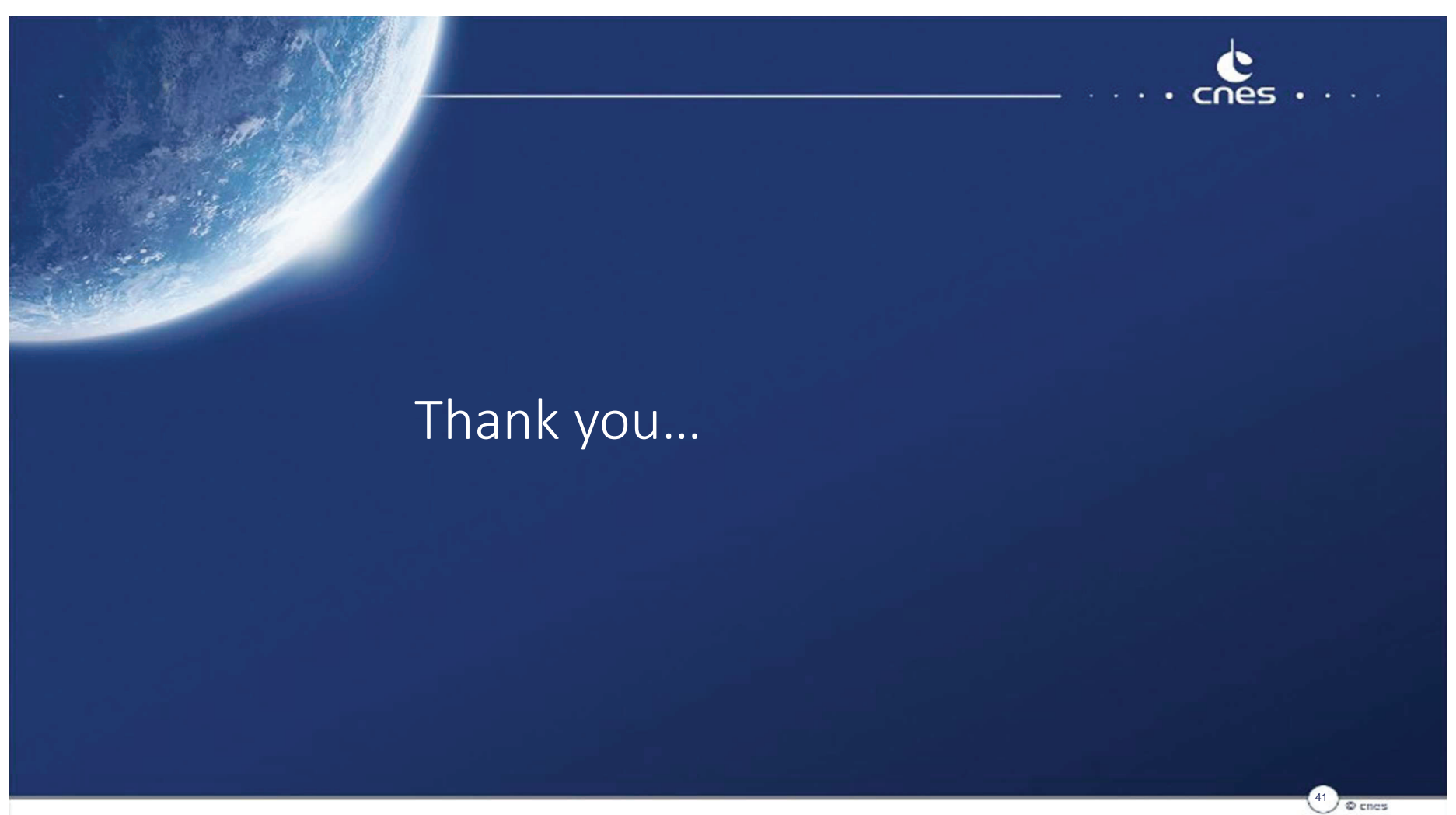

Proc. of SPIE Vol. 11852 1185204-22

Downloaded From: https://www.spiedigitallibrary.org/conference-proceedings-of-spie on 26 Apr 2023 Terms of Use: https://www.spiedigitallibrary.org/terms-of-use 